\title{
Research on Quality Management Evaluation Method of Intelligent Robot Upgrade and R\&D Project
}

\author{
WU Tian-qi ${ }^{1 s t a}$, YAO Lu ${ }^{2 n d}$ \\ ${ }^{1}$ Department of Management engineering and equipment economics Naval University of Engineering Wuhan, China
}

\begin{abstract}
In order to promote the high-quality development of intelligent robot upgrade R\&D projects, promote the progress of social and economic intelligence, and improve production efficiency, a dynamic weighted evaluation method based on network cohesion of balanced groups is proposed. This method is based on balanced grouping of evaluation groups to ensure the relative homogeneity of evaluation groups. It uses dynamic interactive evaluation to construct a network adjacency matrix based on the consistency of pairwise evaluations within the group. And it collects Horizontal information based on the concept of network cohesion. A stage planning function is constructed to calculate the weights of multiple rounds of evaluation, Finally, a comprehensive evaluation result is obtained after vertical merging. This paper provides a new evaluation method for improving the quality management of intelligent robot upgrade R\&D projects.
\end{abstract}

\section{Introduction}

As the economy continues to develop, intelligent robots have entered thousands of households, and all walks of life have improved production efficiency thanks to the assistance of intelligent robots. According to different actual needs, the functions of intelligent robots are becoming more and more complex, meanwhile, the requirements for intelligent robot manufacturers are gradually increasing. The upgrading and research and development projects of intelligent robots are independent of intelligent robot manufacturing projects, and they have technology-intensive, many related parties, and people's expectations[1]. Therefore, it is very important for the quality management of intelligent robot upgrade research and development projects.

Group evaluation is an approach commonly used for intelligent robot evaluation, which is mainly based on group homogeneity[2], that is, there is no difference among members participating in the group evaluation, as manifested by uniform scale of experts, consistent knowledge system with the same structure, and similar experiences and backgrounds[3]. Obviously, the assumption of homogeneity will seriously affect the results of evaluation, especially when assessing complicated subjects with a wide range of indicators, where a unified evaluation standard is hardly available. Therefore, it is difficult to ensure homogeneity of groups via simple grouping methods[4].

In view of the above situation, $\mathrm{Su}$ Weihua et al.[5] designed a "medium" and a "bridge" to connect subgroups and reduce the gap between chain evaluation values. Yang Lei et al.[6] studied the effect of interaction mechanism on the views of a group, compared and analyzed opinions and comments among groups. Su Weihua et al.[7] improved the consistency of evaluation based on the assessment of conflicts in the evaluation. However, studies like these need to be designed before the evaluation begins, which requires the organizers to be familiar with the attributes of the subjects and experts participating in the evaluation, making it difficult to perform in real scenarios[8]. Based on this, this paper maintained a balance when dividing expert into groups based on dynamic weighted interactive evaluation to ensure accurate and scientific decisionmaking of groups.

\section{Balanced Group Quality Management and Evaluation Based on Dynamic Weighted Network Cohesion}

Based on the abovementioned analysis, the traditional group evaluation requires evaluators of homogeneity. However, in actual group evaluation, it is difficult to control factors such as personal experience, cognition and subjective criteria in addition to uncertainties in the results of group evaluation, where homogeneity is hardly available. The balanced grouping method aims at identifying the level and grade of evaluators through evaluation results to place evaluators of homogeneity in the same group and ensure overall consistency of a group. After balanced grouping, the adjacency network matrix was constructed based on evaluation results between two individual evaluators, where the latitudinal weight was determined by network cohesion to generate a stageplanning function that will be used to calculate the meridional weight, thus obtaining the final evaluation results of the balanced groups. The logical process involved in quality management and evaluation of 
balanced groups with dynamic weighted network cohesion is shown in Figure 1.

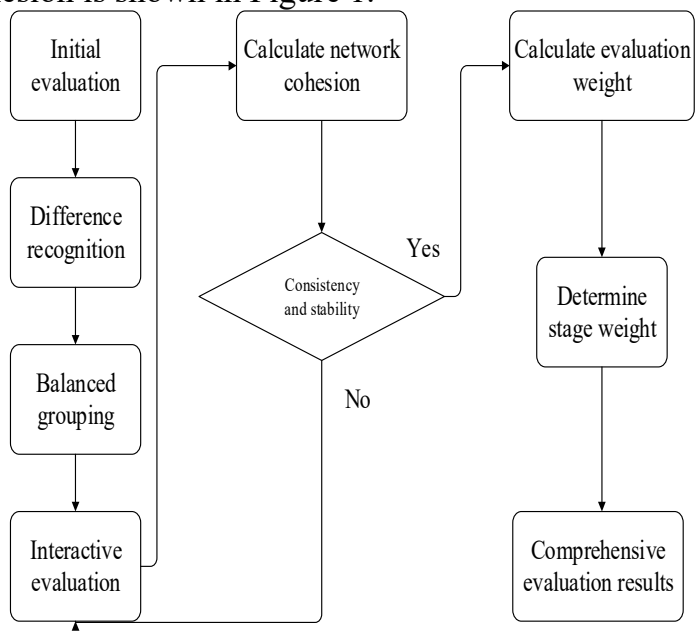

Figure 1 Flow Diagram

In Figure 1, a preliminary assessment of indicators used by evaluation groups serves as the first step to identify differences among individuals of the evaluation groups based on initial evaluation results. Then sort the differences and redistribute individuals for balanced grouping, based on which an interactive evaluation of indicators is accomplished. Through balanced grouping, the equivalence and homogeneity of evaluation groups is improved, thus ensuring greater group consistency.

However, there tend to be differences of individual credibility in group evaluation, which could affect the results of group evaluation. At present, there are two main approaches to deal with such problems, namely feedback and adjustment of individual weight. Feedback mechanism, also known as interactive evaluation mechanism, improves overall consistency of evaluation groups by repeatedly asking individual evaluators to adjust their evaluations [9]. The adjustment mechanism of evaluation weights is accomplished by analyzing differences among individual weights to ensure consistency.

In actual evaluation, the repeated evaluations of one indicator can be integrated as the result of overall evaluation, the consistency of which with the group evaluation results would be further improved in each stage of evaluation. The adjustment mechanism of individual weight is based on single round of evaluation, featuring strong individuality. In this paper, the dynamic weighted network cohesion was included in the interactive evaluation mechanism to construct an adjacency matrix based on the pairwise consistency of individual evaluators. Increased network cohesion and the weight of individuals consistent with group decision, coupled with compromised weight of individuals inconsistent with group decision, would reduce disturbance of specific data on the overall evaluation. Balanced grouping and interactive evaluation based on dynamic weighted network cohesion could improve accuracy of the evaluation results and reduce the influence of collective and individual factors on the evaluation.

\section{Design and Implementation of Balanced Grouping Evaluation Based on Dynamic Weighted Network Cohesion}

\subsection{Establishment of an evaluation group for initial evaluation}

The evaluation indicator set is $X=\left\{x_{r} \mid x_{1}, x_{2}, \cdots, x_{n}\right\}$, and the evaluation group is $E_{i}(i=1,2, \cdots, g)=\left\{e_{s} \mid e_{1}, e_{2}, e_{m}\right\}$. The evaluation result of evaluation indicator $x_{r}$ by individual evaluator $e_{s}$ is $a_{s r}$.

\subsection{Calculate differences between the evaluation results of individuals}

On the basis of the initial evaluation results, the difference of individual $e_{s}$ and other individuals from indicator $x_{r}$ is calculated as: $\mathrm{d}(s, \mathrm{u})$,

$\mathrm{d}(\mathrm{s}, \mathrm{u})=\frac{1}{\mathrm{~m} \times \mathrm{n}} \sum_{s \neq u}^{m} \sum_{\mathrm{r}=1}^{n}\left|a_{s r}-a_{u r}\right|$

where $m$ and $n$ represent the number of individual evaluators and the number of evaluation indicators in the group, $a_{s r}$ indicates the evaluation of evaluation indicator $x_{r}$ by individual evaluator $e_{s}$, and likewise $a_{u r}$ indicates the evaluation of evaluation indicator $x_{r}$ by individual evaluator $e_{u}$.

\section{Balanced grouping}

Redistribute the group members based on differences of individual es from others in terms of evaluation indicator $x_{r}$. Calculate the differences between evaluation results by individuals to form an evaluation difference matrix, based on which the overall evaluation difference between individuals is calculated. Rank the difference, after which the evaluation individuals are equally divided in a balanced manner and denoted as $E_{i}^{\prime}(i=1,2, \cdots, h)$.

\subsection{Interactive evaluation of evaluation indicators by balanced evaluation groups}

After publishing the results of the last round of evaluation, the balanced evaluation group reevaluates the evaluation indicators, after which the consistency between individual $e_{s}$ and individual $e_{u}$ on indicator $x_{r}$ and indicator $x_{o}$ is calculated, which was marked by $C_{r o}^{s u}[10]$ :

$$
C_{r o}^{s u}=\left\{\begin{array}{l}
1, \text { If } a_{s r}<a_{u o} \text { and } a_{s r}<a_{u o}, \text { or } a_{s r}>a_{u o} \text { and } a_{s r}>a_{u o}, \\
\quad \text { or } a_{s r}=a_{u o} \text { and } a_{s r}=a_{u o} ; \\
0.5, \text { if } a_{s r}=a_{u o} \text { and } a_{s r} \neq a_{u o}, \text { or } a_{s r} \neq a_{u o} \text { and } a_{s r}=a_{u o} ; \\
0, \text { else }
\end{array}\right.
$$

In repeated interactive evaluations, the consistency indicator $C_{r o}^{s u}$ is transformed into the similarity weight 
$w_{s u}^{t}$ among individual evaluators. The greater the similarity weight, the closer the evaluation results of individuals.

$$
w_{s u}^{t}=\frac{4 \sum_{r=1}^{n} \sum_{o=r+1}^{n} C_{r o}^{s u}}{n(n-1)}-1
$$

The similarity weight $w_{s u}^{t}$ is processed using formula:

$$
w_{s u}^{t^{*}}=\frac{w_{s u}^{t}+1}{2}
$$

After normalization, the similarity weight adjacency matrix of the $t$-th round of interactive evaluation by individuals is obtained:

$$
w^{t^{*}}=\left[\begin{array}{cccc}
w_{11}^{t^{*}} & w_{12}^{t^{*}} & \ldots & w_{1 m}^{t^{*}} \\
w_{21}^{t^{*}} & w_{22}^{t^{*}} & \ldots & w_{2 m}^{t^{*}} \\
\ldots & \ldots & \ldots & \ldots \\
w_{m 1}^{t^{*}} & w_{m 2}^{t^{*}} & \ldots & w_{m m}^{t^{*}}
\end{array}\right] .
$$

\subsection{Calculate network cohesion $\partial\left(\mathrm{WJ}_{t}\right)$}

In the t-th round of interactive evaluation, the importance of individuals participating in the evaluation is determined by the sum of other individual evaluations and their similarity weights [11], that is, $q_{s}^{t}=\sum w_{s u}^{t^{*}}-1$, when the average weights of the whole interactive network total

$$
Q^{t}=\sum_{s=1}^{m} \frac{q_{s}^{t}}{m-1} .
$$

The distance between individuals $d_{s u}^{t}$ is expressed by the reciprocal of similarity weights, that is,

$$
d_{s u}^{t}=\left(w_{s u}^{t^{*}}\right)^{-1} \text {. }
$$

The average distance between individuals in the interactive network is calculated by

$$
\overline{\mathrm{D}}^{t}=\frac{\sum d_{s u}^{t}-m}{m(m-1)} \text {. }
$$

The network cohesion $\partial\left(\mathrm{WJ}_{t}\right)$ is expressed by the reciprocal of the sum of average weight times average distance [12]:

$$
\partial\left(\mathrm{WJ}_{t}\right)=\left(Q^{t} \times \bar{D}^{t}\right)^{-1} .
$$

\subsection{Determine the conditions of interactive evaluation termination}

The change of network cohesion $\partial\left(\mathrm{WJ}_{t}\right)$ could determine the stability of evaluation $\lambda^{t}$. When

$$
\lambda^{t}=\left|1-\frac{\partial\left(\mathrm{W} J_{t}\right)}{\partial\left(\mathrm{W} J_{t-1}\right)}\right|<\varepsilon .
$$

the result of interactive evaluation is considered stable. The consistency of evaluation $\theta^{t}$ is determined according to the average distance between individual evaluators and deviation in average distance of the network. When

$$
\theta^{t}=\frac{1}{m} \sqrt{\sum_{u=1}^{m}\left(\frac{1}{m-1} \sum_{s=1}^{m}\left(d_{s u}^{t}-1\right)-\bar{D}^{t}\right)^{2}}<\varphi .
$$

the results of interactive evaluation are considered consistent. When and only when the interactive evaluation results are stable and consistent, that is, when $\lambda^{t}$ and $\theta^{t}$ meet the conditions, the interaction would be terminated. Both $\varepsilon$ and $\varphi$ are preset thresholds.

\subsection{Calculate the importance of evaluation result $I\left(a_{s}^{t}\right)$}

The $\partial\left(\mathrm{WJ}_{t}\right)$ represents the network cohesion of interactive evaluation at the $\mathrm{t}$-th round, and $\partial\left(\mathrm{WJ}_{t} \bullet a_{s}^{t}\right)$ marks the network cohesion of interactive evaluation at the $\mathrm{t}$-th round when evaluations by individual es are removed. The importance of individual evaluation $I\left(a_{s}^{t}\right)$ is calculated by

$$
I\left(a_{s}^{t}\right)=1-\frac{\partial\left(\mathrm{W} J_{t}\right)}{\partial\left(\mathrm{W} J_{t} \bullet a_{s}^{t}\right)} .
$$

\subsection{Calculate the overall evaluation results of a single round of evaluation $a_{r}^{t^{*}}$}

After normalizing the importance of the evaluation results of each individual $I\left(a_{s}^{t}\right)$, the evaluation weight of individuals $w_{s}^{t}$ in this round is obtained by

$$
w_{s}^{t}=\frac{I\left(a_{s}^{t}\right)}{\sum_{s=1}^{m} I\left(a_{s}^{t}\right)}
$$

Then, the overall evaluation result of the $n$-th evaluation indicator at the $\mathrm{t}$-th round is given by

$$
a_{r}^{t^{*}}=w_{s}^{t} \bullet a_{s r}^{t}
$$

The overall evaluation results of each round form the matrix of interactive evaluation results

$$
A^{t^{*}}=\left[\begin{array}{cccc}
a_{1}^{1^{*}} & a_{2}^{1^{*}} & \ldots & a_{n}^{1^{*}} \\
a_{1}^{2^{*}} & a_{2}^{2^{*}} & \ldots & a_{n}^{2^{*}} \\
\ldots & \ldots & \ldots & \ldots \\
a_{1}^{l^{*}} & a_{2}^{l^{*}} & \ldots & a_{n}^{l^{*}}
\end{array}\right] .
$$

\subsection{Determine the weight at the interactive stage $\beta^{t}$}

The continuous interactions would gradually reduce fluctuations of evaluation results from individuals while increasing the stage weight. Meanwhile, considering the evaluation results of individuals are closest to the overall evaluation results of the same round, the planning function is constructed as [13]: 


$$
\left\{\begin{array}{l}
\min \sum_{t=1}^{l} \beta_{r}^{t} \sum_{s=1}^{m}\left(a_{s r}^{t}-a_{r}^{t^{*}}\right)^{2} \\
\text { s.t. } \beta_{r}^{t}-\beta_{r}^{t+1} \leq 0 \\
\sum_{t=1}^{l} \beta_{r}^{t}=1 \\
\beta_{r}^{t} \geq 0
\end{array} .\right.
$$

Solve the stage weight of each individual, then we will have the stage weight matrix:

$$
\mathrm{B}^{\mathrm{t}}=\left[\begin{array}{cccc}
\beta_{1}^{1} & \beta_{2}^{1} & \ldots & \beta_{1}^{1} \\
\beta_{1}^{2} & \beta_{2}^{2} & \ldots & \beta_{\mathrm{n}}^{2} \\
\ldots & \ldots & \ldots & \ldots \\
\beta_{1}^{1} & \beta_{2}^{1} & \ldots & \beta_{\mathrm{n}}^{1}
\end{array}\right] .
$$

\subsection{Calculate the overall evaluation performance $P_{r}$ of balanced groups}

$$
p_{r}^{i}=a_{r}^{t^{*}} \bullet \beta_{r}^{t}
$$

from which the overall evaluation result of the i-th balanced group is obtained: $\left[p_{r}^{i}\right]=\left\lfloor p_{1}^{i}, p_{2}^{i}, \ldots, p_{n}^{i}\right\rfloor$

As all groups are divided in a balanced manner, the arithmetic average is used to calculate the overall evaluation performance $P_{r}$ of the balanced groups,

$$
P_{r}=\sum_{i=1}^{g} p_{r}^{i}
$$

\section{Application of Balanced Group Evaluation Based on Dynamic Weighted Network Cohesion}

To evaluate the quality management maturity of an intelligent robot $R \& D$ enterprise through an intelligent robot upgrade and $R \& D$ project, a quality evaluation team was set up, consisting of six experts of quality management, six project team members and six clients respectively. The evaluation system is shown in TABLE 1. The evaluation members scored 1-100 points on the indicators, where 20 points, 40 points, 60 points, 80 points and 100 points represented very poor, poor, fair, good, excellent. The closer the score to a specific level, the more likely it would be included in the corresponding category. Due to limitation of space, this paper demonstrated the indicators of quality management expertise as an example.

Table1. Quality Management Maturity Indicators for intelligent robot upgrade and $R \& D$ projects

\begin{tabular}{|c|c|c|c|c|}
\hline \multirow{2}{*}{ Dimension } & Weights & index & $\begin{array}{c}\text { Index } \\
\text { Weight }\end{array}$ & $\begin{array}{c}\text { Total } \\
\text { weight }\end{array}$ \\
\hline \multirow{2}{*}{ Finance } & \multirow{2}{*}{$4707 \%$} & $X_{1}:$ Roe & $17.84 \%$ & $8.40 \%$ \\
\cline { 3 - 5 } & \multirow{2}{*}{$X_{2}:$ Sales margin } & $13.29 \%$ & $6.26 \%$ \\
\cline { 3 - 5 } & & $\begin{array}{c}X_{3}: \text { Operating profit } \\
\text { margin }\end{array}$ & $10.96 \%$ & $5.16 \%$ \\
\hline
\end{tabular}

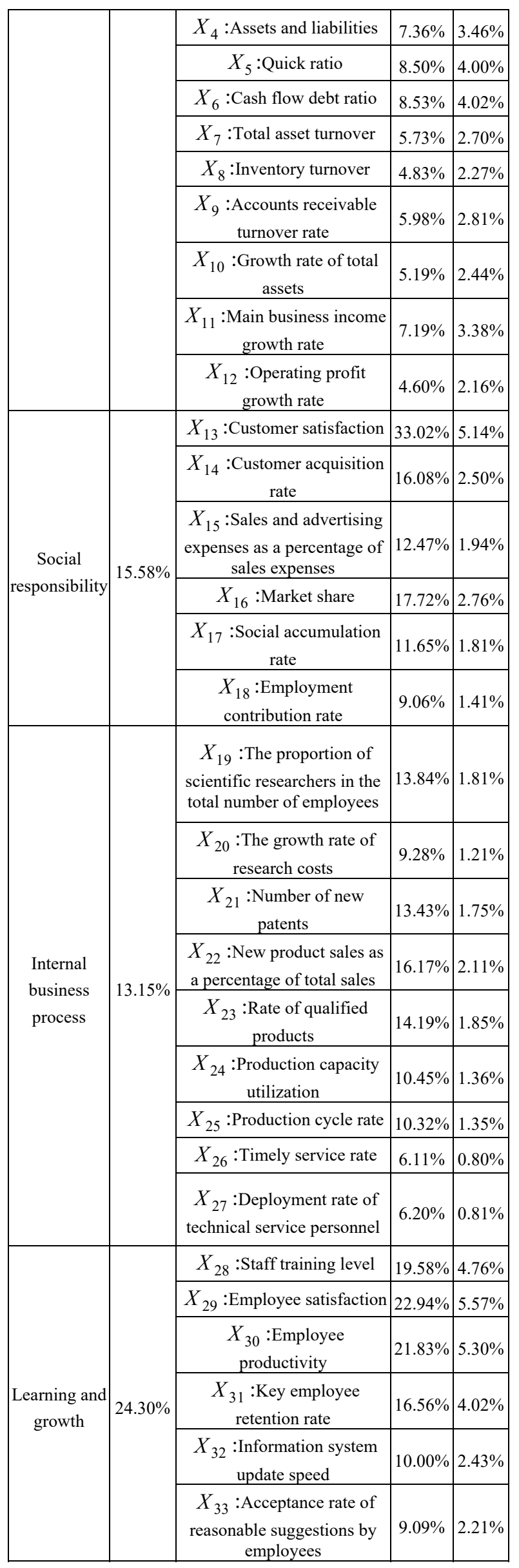




\subsection{Initial evaluation}

The evaluation members were divided into three groups according to their sources, which were named $E_{1}, E_{2}$ and $E_{3}$, representing quality management experts, project team members and clients respectively. They were asked to score indicators of Learning and growth, the results of which are shown in TABLE 2.

Table2. Initial Scoring Results

\begin{tabular}{|c|c|c|c|c|c|c|c|}
\hline Group & $\begin{array}{c}\text { Index } \\
\text { member }\end{array}$ & $X_{28}$ & $X_{29}$ & $X_{30}$ & $X_{31}$ & $X_{32}$ & $X_{33}$ \\
\hline \multirow{6}{*}{$E_{1}$} & $e_{1}$ & 79 & 72 & 53 & 67 & 63 & 72 \\
\hline & $e_{2}$ & 81 & 75 & 57 & 66 & 66 & 73 \\
\hline & $e_{3}$ & 82 & 84 & 78 & 82 & 86 & 78 \\
\hline & $e_{4}$ & 84 & 69 & 61 & 74 & 76 & 82 \\
\hline & $e_{5}$ & 89 & 83 & 77 & 82 & 74 & 73 \\
\hline & $e_{6}$ & 74 & 77 & 82 & 77 & 81 & 79 \\
\hline \multirow{6}{*}{$E_{2}$} & $e_{7}$ & 93 & 90 & 81 & 85 & 68 & 85 \\
\hline & $e_{8}$ & 79 & 74 & 75 & 72 & 57 & 92 \\
\hline & $e_{9}$ & 85 & 89 & 88 & 93 & 74 & 95 \\
\hline & $e_{10}$ & 94 & 93 & 90 & 77 & 65 & 91 \\
\hline & $e_{11}$ & 93 & 96 & 95 & 90 & 91 & 97 \\
\hline & $e_{12}$ & 95 & 97 & 89 & 92 & 87 & 95 \\
\hline \multirow{6}{*}{$E_{3}$} & $e_{13}$ & 68 & 74 & 83 & 74 & 83 & 82 \\
\hline & $e_{14}$ & 77 & 84 & 85 & 82 & 87 & 88 \\
\hline & $e_{15}$ & 80 & 77 & 86 & 73 & 76 & 73 \\
\hline & $e_{16}$ & 94 & 93 & 74 & 88 & 92 & 83 \\
\hline & $e_{17}$ & 73 & 74 & 77 & 82 & 88 & 92 \\
\hline & $e_{18}$ & 74 & 74 & 79 & 73 & 78 & 76 \\
\hline
\end{tabular}

\subsection{Balanced grouping}

According to the results of initial evaluation, the difference between individual evaluations was calculated, as shown in TABLE3. Then, they were regrouped using balanced grouping method into Major Difference Group $E_{1}^{\prime}\left(e_{1}, e_{2}, e_{8}, e_{10}, e_{11}, e_{12}\right)$, Medium Difference Group $E_{2}^{\prime}\left(e_{4}, e_{7}, e_{9}, e_{13}, e_{16}, e_{17}\right)$, and Minor Difference Group $E_{3}^{\prime}\left(e_{3}, e_{5}, e_{6}, e_{14}, e_{15}, e_{18}\right)$. After regrouping, the balanced groups reevaluated the indicators, and the secondary evaluation results are shown in TABLE4. By comparing the evaluation results before and after regrouping, it was found that individuals whose result in the first round was highly different with the group evaluation showed much less inconsistency in the second evaluation. The evaluation results of individuals tended to be more consistent, proving the benefits of balanced grouping.
Table3. Evaluation of the degree of individual differences

\begin{tabular}{|c|c|}
\hline Evaluation of individuals & Degree of difference \\
\hline$e_{1}$ & 41.00 \\
\hline$e_{11}$ & 40.72 \\
\hline$e_{2}$ & 38.22 \\
\hline$e_{12}$ & 37.50 \\
\hline$e_{8}$ & 33.56 \\
\hline$e_{10}$ & 33.50 \\
\hline$e_{4}$ & 32.72 \\
\hline$e_{16}$ & 32.67 \\
\hline$e_{9}$ & 30.44 \\
\hline$e_{13}$ & 29.78 \\
\hline$e_{17}$ & 29.11 \\
\hline$e_{7}$ & 28.44 \\
\hline$e_{18}$ & 27.50 \\
\hline$e_{15}$ & 26.39 \\
\hline$e_{5}$ & 26.22 \\
\hline$e_{14}$ & 26.06 \\
\hline$e_{6}$ & 25.33 \\
\hline$e_{3}$ & 25.28 \\
\hline
\end{tabular}

Table4. Secondary Scoring Results

\begin{tabular}{|c|c|c|c|c|c|c|c|}
\hline Group & member Index & $X_{28}$ & $X_{29}$ & $X_{30}$ & $X_{31}$ & $X_{32}$ & $X_{33}$ \\
\hline \multirow{6}{*}{$E_{1}^{\prime}$} & $e_{1}$ & 83 & 79 & 72 & 76 & 69 & 82 \\
\hline & $e_{2}$ & 84 & 83 & 73 & 74 & 68 & 82 \\
\hline & $e_{8}$ & 85 & 82 & 77 & 77 & 67 & 88 \\
\hline & $e_{10}$ & 88 & 88 & 80 & 77 & 70 & 87 \\
\hline & $e_{11}$ & 89 & 88 & 83 & 80 & 80 & 88 \\
\hline & $e_{12}$ & 88 & 88 & 80 & 85 & 80 & 90 \\
\hline \multirow{6}{*}{$E_{2}^{\prime}$} & $e_{4}$ & 85 & 78 & 73 & 79 & 78 & 82 \\
\hline & $e_{7}$ & 84 & 85 & 82 & 85 & 78 & 88 \\
\hline & $e_{9}$ & 79 & 78 & 83 & 78 & 78 & 80 \\
\hline & $e_{13}$ & 84 & 79 & 81 & 84 & 80 & 78 \\
\hline & $e_{16}$ & 83 & 84 & 78 & 84 & 82 & 83 \\
\hline & $e_{17}$ & 78 & 78 & 80 & 78 & 80 & 80 \\
\hline \multirow{6}{*}{$E_{3}^{\prime}$} & $e_{3}$ & 85 & 83 & 78 & 80 & 84 & 82 \\
\hline & $e_{5}$ & 85 & 85 & 80 & 82 & 80 & 82 \\
\hline & $e_{6}$ & 83 & 83 & 82 & 83 & 82 & 80 \\
\hline & $e_{14}$ & 86 & 84 & 80 & 80 & 78 & 85 \\
\hline & $e_{15}$ & 83 & 83 & 83 & 80 & 82 & 85 \\
\hline & $e_{18}$ & 79 & 82 & 78 & 79 & 84 & 87 \\
\hline
\end{tabular}

\subsection{Interactive evaluation on evaluation indicators by balanced evaluation group}

Given $\varepsilon=0.001$ and $\varphi=0.15$, the interactive evaluation by $E_{1}^{\prime}, E_{2}^{\prime}$ and $E_{3}^{\prime}$ terminated at round 6, 
5 and 3 respectively. The changes of $\varepsilon$ and $\varphi$ are shown in TABLE 5. Take the balanced group of $E_{3}^{\prime}$ as an example, the evaluation result of which at each round is shown in TABLE 6.

Table5. Changes of $\varepsilon$ and $\varphi$ in Each Round

\begin{tabular}{|c|c|c|c|c|c|c|}
\hline \multirow{2}{*}{ Round } & \multicolumn{2}{|c|}{$E_{1}^{\prime}$} & \multicolumn{2}{c|}{$E_{2}^{\prime}$} & \multicolumn{2}{|c|}{$E_{3}^{\prime}$} \\
\cline { 2 - 7 } & $\varepsilon$ & $\varphi$ & $\varepsilon$ & $\varphi$ & $\varepsilon$ & $\varphi$ \\
\hline $\mathbf{0}$ & 0.000 & 0.030 & 0.000 & 0.057 & 0.000 & 0.088 \\
\hline $\mathbf{1}$ & 0.023 & 0.033 & -0.002 & 0.061 & 0.027 & 0.064 \\
\hline $\mathbf{2}$ & -0.006 & 0.040 & 0.005 & 0.059 & 0.015 & 0.060 \\
\hline $\mathbf{3}$ & 0.052 & 0.039 & -0.009 & 0.057 & 0.001 & 0.060 \\
\hline $\mathbf{4}$ & -0.002 & 0.036 & -0.003 & 0.038 & & \\
\hline $\mathbf{5}$ & 0.003 & 0.035 & -0.000 & 0.041 & & \\
\hline $\mathbf{6}$ & -0.001 & 0.036 & & & & \\
\hline
\end{tabular}

Table6. Interactive Evaluation Results of Each Round of E3 Balanced Group

\begin{tabular}{|c|c|c|c|c|c|c|c|c|}
\hline Rounds & Group & $\begin{array}{l}\text { Group } \\
\text { member }\end{array}$ & $X_{28}$ & $X_{29}$ & $X_{30}$ & $X_{31}$ & $X_{32}$ & $X_{33}$ \\
\hline \multirow{6}{*}{$\mathbf{t}=\mathbf{0}$} & \multirow{6}{*}{$E_{3}^{\prime}$} & $e_{3}$ & 82 & 84 & 78 & 82 & 86 & 78 \\
\hline & & $e_{5}$ & 89 & 83 & 77 & 82 & 74 & 73 \\
\hline & & $e_{6}$ & 74 & 77 & 82 & 77 & 81 & 79 \\
\hline & & $e_{14}$ & 77 & 77 & 77 & 77 & 77 & 77 \\
\hline & & $e_{15}$ & 80 & 77 & 86 & 73 & 76 & 73 \\
\hline & & $e_{18}$ & 74 & 74 & 79 & 73 & 78 & 76 \\
\hline \multirow{6}{*}{$t=1$} & \multirow{6}{*}{$E_{3}^{\prime}$} & $e_{3}$ & 85 & 83 & 78 & 80 & 84 & 82 \\
\hline & & $e_{5}$ & 85 & 85 & 80 & 82 & 80 & 82 \\
\hline & & $e_{6}$ & 83 & 83 & 82 & 83 & 82 & 80 \\
\hline & & $e_{14}$ & 77 & 84 & 85 & 82 & 87 & 88 \\
\hline & & $e_{15}$ & 83 & 83 & 83 & 80 & 82 & 85 \\
\hline & & $e_{18}$ & 79 & 82 & 78 & 79 & 84 & 87 \\
\hline \multirow{6}{*}{$t=2$} & \multirow{6}{*}{$E_{3}^{\prime}$} & $e_{3}$ & 83 & 83 & 81 & 80 & 84 & 82 \\
\hline & & $e_{5}$ & 83 & 84 & 81 & 81 & 83 & 83 \\
\hline & & $e_{6}$ & 83 & 83 & 82 & 82 & 82 & 82 \\
\hline & & $e_{14}$ & 82 & 84 & 82 & 81 & 83 & 83 \\
\hline & & $e_{15}$ & 83 & 83 & 81 & 81 & 83 & 83 \\
\hline & & $e_{18}$ & 81 & 83 & 80 & 81 & 84 & 83 \\
\hline \multirow{6}{*}{$\mathbf{t}=\mathbf{3}$} & \multirow{6}{*}{$E_{3}^{\prime}$} & $e_{3}$ & 83 & 83 & 81 & 80 & 84 & 82 \\
\hline & & $e_{5}$ & 83 & 84 & 81 & 81 & 83 & 83 \\
\hline & & $e_{6}$ & 83 & 83 & 82 & 82 & 82 & 82 \\
\hline & & $e_{14}$ & 82 & 84 & 82 & 81 & 83 & 83 \\
\hline & & $e_{15}$ & 83 & 83 & 81 & 81 & 83 & 83 \\
\hline & & $e_{18}$ & 82 & 83 & 80 & 81 & 84 & 83 \\
\hline
\end{tabular}

\subsection{Calculate the evaluation weight of individuals in each round}

Calculate pairwise consistency coefficient based on the evaluation results of each round using Formula (2), which is then converted into similarity weight matrix by Formula (3) and (4). The network cohesion is calculated by
Formula (5)-(8). Thus, the evaluation weights of each round of individual evaluation could be obtained by Formula (11) and (12), from which the overall evaluation result of balanced groups in each round is derived. The overall evaluation results of the balanced evaluation group $E_{3}^{\prime}$ in each round are shown in TABLE 7.

Table7. Overall Evaluation Values of E3 Balanced Evaluation Group in Each Round

\begin{tabular}{|c|c|c|c|c|c|c|}
\hline $\begin{array}{c}\text { Index } \\
\text { Rounds }\end{array}$ & $X_{28}$ & $X_{29}$ & $X_{30}$ & $X_{31}$ & $X_{32}$ & $X_{33}$ \\
\hline $\mathrm{t}=0$ & 81.45 & 82.25 & 80.38 & 82.18 & 80.90 & 83.29 \\
\hline $\mathrm{t}=1$ & 82.65 & 81.56 & 80.31 & 80.96 & 81.02 & 83.11 \\
\hline $\mathrm{t}=2$ & 82.17 & 81.17 & 80.54 & 80.69 & 81.03 & 83.26 \\
\hline $\mathrm{t}=3$ & 83.30 & 82.97 & 78.79 & 80.00 & 78.24 & 84.35 \\
\hline
\end{tabular}

\subsection{Calculate overall evaluation results}

Formula (14) is constructed,

$$
\mathrm{B}^{\mathrm{E}_{3}^{\prime}}=\left[\begin{array}{cccccc}
0 & 0 & 0 & 0 & 0 & 0 \\
0 & 0 & 0 & 0 & 1 / 3 & 0 \\
0.149 & 0 & 1 / 2 & 1 / 2 & 1 / 3 & 1 / 2 \\
0.851 & 1 & 1 / 2 & 1 / 2 & 1 / 3 & 1 / 2
\end{array}\right]
$$

based on which the weight matrix of $E_{3}^{\prime}$ Balanced Group was obtained for the calculation of the overall evaluation results of the group. The final results were derived by calculating arithmetic mean values of Balanced Group $E_{1}^{\prime}, E_{2}^{\prime}$ and $E_{3}^{\prime}$, as shown in TABLE 8.

Table8. Overall Evaluation Results

\begin{tabular}{|c|c|c|c|c|c|c|}
\hline Group & $X_{28}$ & $X_{29}$ & $X_{30}$ & $X_{31}$ & $X_{32}$ & $X_{33}$ \\
\hline$E_{1}^{\prime}$ & 84.43 & 84.18 & 78.28 & 80.46 & 74.37 & 85.06 \\
\hline$E_{2}^{\prime}$ & 83.13 & 80.76 & 78.88 & 80.38 & 77.83 & 84.73 \\
\hline$E_{3}^{\prime}$ & 83.13 & 82.97 & 79.67 & 80.35 & 80.09 & 83.81 \\
\hline Comprehensive value & 83.13 & 82.97 & 79.28 & 80.35 & 80.09 & 83.81 \\
\hline
\end{tabular}

\subsection{Calculate the overall evaluation values of the indicator system}

Calculate the overall evaluation values of all indicators using the abovementioned formula, which, combined with indicator weight, could obtain the overall evaluation of the indicator evaluation system.

\section{Conclusion}

Based on the quality management system of intelligent robots upgrading and research and development projects, this paper designed a balanced group evaluation method based on dynamic weighted network cohesion with the following characteristics:

Unlike traditional grouping of experts participating in 
the evaluation, this paper regrouped experts based on the balance theory to reduce cognitive differences among experts of the same group, improving consistency and credibility of the evaluation.

In interactive evaluation, the evaluation results of the same group were publicized before each round to reduce inconsistency between individuals of the group. The application of network cohesion and similarity weight of experts made the weight adjustment more intuitive and accessible to individuals.

The determination of stage weights added practicality to the evaluation. The incorporation of evaluation results of subjects into the calculation of overall evaluation values improved accuracy and credibility of the evaluation.

Due to limitation of space, this paper simplified procedures involved in the study, and found defects of the investigation as the participants were asked to repeatedly evaluate various indicators of the same subject, thus lowering the credibility of the evaluation. In the meantime, the excessive scale of participants and indicators would hamper the prediction of the evaluation results, imposing challenges to the organization of the evaluation due to increased cost and lower efficiency, which needs further study in the future.

\section{References}

1. Robotics; New Robotics Study Findings Recently Were Reported by Researchers at Chinese University of Hong Kong (Toward "On-Demand" Materials Synthesis and Scientific Discovery through Intelligent Robots)[J]. Journal of Robotics \& Machine Learning,2020.

2. Weihua Su, Chonghui Zhang, Shouzhen Zeng. Uncertain Induced Heavy Aggregation Distance Operator and its Application to Decision Making[J]. Cybernetics and Systems, 2015, 46(3-4).

3. Soutschek Alexander, Schubert Torsten. Dynamic adjustments of cognitive control during economic decision making.[J]. Acta psychologica,2014,152.

4. Michelle Baddeley, Sophia Parkinson. Group decision-making: An economic analysis of social influence and individual difference in experimental juries[J]. Journal of Socio-Economics, 2012, 41(5).

5. Su Weihua, Zhang Chonghui, Zeng Shouzhen. Design of chain evaluation mechanism based on information entropy theory[J]. Statistical Research, 2014, 31(2): 85-90.

6. Yang Lei, Guo Min. Opinion convergence analysis of interaction among different subgroups in dynamic group decision making[J]. Industrial Engineering Journal, 2011, 14(4): 104-109.

7. Su Weihua, Zhang Chonghui, Zeng Shouzhen. A dynamic group evaluation method with variable subjects and its application[J]. Statistical Research, 2015, 32(7): 100-105.

8. Zhang Chonghui, Su Weihua, Zeng Shouzhen. Design and application of group evaluation mechanism based on equilibrium theory[J]. Statistical Research, 2018,
35(1): 82-90.

9. Su Weihua, Zhang Chonghui. The design of two-stage sub-group evaluation mechanism[J]. Systems Engineering-Theory Practice, 2016, 36(3): 760-767.

10. Li Yongli, Wu Chong, Zhang Xiaofei. Evaluator's weight distribution method considering the effect of network interaction[J]. Journal of Management Science in China, 2016, 19(4): 32-44.

11. MENG Y, LI T.Cooperative Stabilization of Linear Multi-Agent Systems with Limited Communication Data Rate[C]//11 th IEEE International Conference on Control\&Automation(ICCA). Taichuang: IEEE, 2014: 1 186-1 191.

12. Zhu Tao, Zhang Shuiping, Guo Rongxiao, et al. Improved evaluation method for node importance based on node contraction in weighted complex networks[J]. Systems Engineer ing and Electr onics, 2009, 31(8): 1902-1905.

13. Zhang Faming, Xiong Jieni. Interactive evaluation information aggregation method based on dynamic weighted network cohesion[J]. Chinese Journal of Management, 2018, 15(8)1231-1239. 